\title{
Robots Visit Homes For Elderly People Who Have Difficulty Going Out and Practice Brain Training
}

\author{
Kazue Sawami, Mitsuo Kimura, Tetsuro Kitamura, Masahiko Kawaguchi, \\ Mihoko Furusumi, Chizuko Suishu, Naoko Morisaki, and Sonomi Hattori
}

\begin{abstract}
Introduction: When it becomes difficult for the elderly to go out due to a decrease in walking ability, the decline in their mental and physical function accelerates. Currently, elderly people who have difficulty going out have no effective measures in place. Therefore, we wanted to prevent their functional decline by visiting homes and intervening. For the intervention, a robot developed for brain training for the elderly people was used. The goal was not only brain training, but also to obtain psychological activation through interaction with robots and supporters.
\end{abstract}

Method: The subjects were elderly people who had difficulty going out. Researchers, supporters, and robot visited their homes to conduct brain training by robots and recollection methods were performed with supporters. Cognitive tests (immediate and delayed reproduction of 10 words) and psychological surveys (interviews) were conducted before and after the intervention.

Result: There were 29 subjects, 8 males, and 21 females, with an average age of $79.5 \pm 4.95$. The maximum score for the cognitive test is 10 . Immediate playback was improved from 5.5 points to 8.1 points $(p<0.01)$, and delayed playback was improved from 4.7 points to 7.5 points $(p<0.01)$. In the analysis of psychological verbatim record, as a result of the preinterview, "Once I stop going out, it becomes troublesome to get dressed up and go out", "There are days when I don't talk about anything all day", "If someone doesn't come or make a phone call, there are no chance of a conversation". In the interview after the start, there were most categories as "I came to think that vitality is important", "I became more motivated", and "I'm looking forward to talking". As for robots, there were many reactions such as cuteness, healing, and fun compared to interpersonal communication.

Conclusion: Brain training during the visits showed that there is a significant improvement in cognitive score, and that it is effective as a countermeasure for the elderly who cannot go out. In addition, the robot has a high affinity for all subjects, the effect of softening the tension associated with receiving the visit was confirmed. Conversation with a robot that resolves the shortage of human resources is meaningful because in the situation where no one talks to anyone throughout the day the utilization of robot shows the improvement in energy and motivation due to increased conversation. By supplementing the interaction with regular supporters to this, it can be a more effective measure.

Index Terms - robots, home-visit, brain-training.

Published on February 08, 2020.

Kazue Sawami, Mitsuo Kimura, Tetsuro Kitamura, Masahiko

Kawaguchi, Mihoko Furusumi are with Nara Medical University, Japan

(corresponding e-mail: sawami@naramed-u.ac.jp).

Chizuko Suishu, Shubun University, Japan.

Naoko Morisaki, Himeji University, Japan.

Sonomi Hattori, Wakayama Medical University, Japan.

\section{INTRODUCTION}

When it becomes difficult for the elderly to go out due to a decrease in walking ability, the decline in their mental and physical function accelerates. Currently, elderly people who have difficulty going out have no effective measures in place. Therefore, we wanted to prevent their functional decline by visiting homes and intervening. For the intervention, a robot developed for brain training for the elderly people was used.

This robot is a humanoid robot that enhances communication functions and aims to increase psychological enjoyment through conversation and to improve thinking ability. This robot is equipped with brain training games and memory recall methods, and is expected to improve cognitive functions. One problem for elderly people who have difficulties going out is that they have significantly less opportunities for interpersonal communication and that various things become a bother for them. About $40 \%$ of elderly people who live alone in Japan have less than one conversation in two to three days [1].

This is a major issue, because conversation activates many areas of the brain. Regarding the activation of many areas of the brain by conversation, not only the language center, but also the frontal motor cortex works to move the mouth and tongue, and the auditory area of the temporal lobe works to listen to people [2]. Talking with someone as much as possible is recommended to prevent dementia, but elderly people who do not go out have very few opportunities for conversation, so that talking with a robot might be able to assist with solving this problem.

The brain training games and reminiscence method methods that can be done together with the robots are based on topics that evoke feelings of nostalgia and something with attachment in elderly people, which is said to make people feel relaxed and comfortable [3,4]. In addition, the brain training games include dual-tasks, such as performing different movements with the left and right side of the body, and delayed recall tasks, in which things that were memorized need to be reproduced later. Dual-tasks stimulate and activate the frontal lobe, which controls movement and thinking, by performing two actions simultaneously and thereby aim to improve cognitive function. Delayed recall tasks are used to discriminate between normal and mild cognitive impairment, and since it is among the first abilities to decrease, aim to improve cognitive function by providing training to strengthen this.

Previous studies about dual-tasks have confirmed that they activate the prefrontal cortex [5-7], and delayed recall 
tasks have been reported to improve intelligence test scores and increase dopamine levels [8,9]. In this intervention, researchers therefore visit the homes of elderly people with the robot, the participants to perform dual-tasks, delayed recall tasks, and reminiscence method, and then measure the improvement of their cognitive function. Another goal is to activate psychologically through conversation with the robot.

\section{METHOD}

\section{A. Subjects}

Elderly people who had difficulty going out.

\section{B. Intervention Method}

Researchers, supporters, and robot visited their homes to conduct brain training by robots and recollection methods were performed with supporters, as shown in Figure 1 and 2.

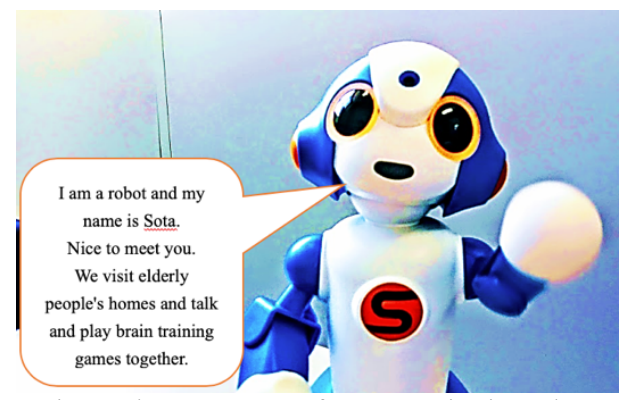

Fig. 1. The appearance of a communication robot

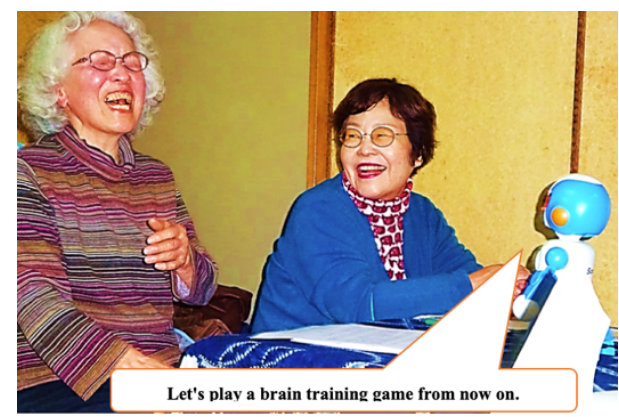

Fig. 2. Robot visits home and trains brain

\section{Evaluation Method}

For the cognitive function test, a ten-word memory test measured immediate memory and recall delayed memory, each 10-point scale.

For psychological surveys, interviews were conducted with the subjects.

\section{Analysis Method}

To compare the scores of immediate memory and recall delayed memory before and after the intervention, the corresponding t-test was carried out.

The results of the interviews were analyzed qualitatively.

\section{E. Ethical Considerations}

The outline of the research, voluntary nature of participation, anonymity, and agreement regarding the publication of the document were explained to prospective participants both in writing and verbally, and their consent was subsequently obtained.
The study protocol was approved by the ethical review board of Nara Medical University.

\section{F. Clinical Trial Registration}

This study has been registered in the clinical trial registration database: University Hospital Medical Information Network (UMIN); registration number: UMIN000037209.

\section{RESUlts}

There were 29 subjects, 8 males, and 21 females, with an average age of $79.5 \pm 4.95$.

The results of cognitive tests: The maximum score for the cognitive test is 10 . Immediate playback was improved from 5.5 points to 8.1 points $(\mathrm{p}=0.000)$, and delayed playback was improved from 4.7 points to 7.5 points $(p=0.000)$.

Result of interview: In the analysis of psychological verbatim record, as a result of the pre-interview, "Once I stop going out, it becomes troublesome to get dressed up and go out", "There are days when I don't talk about anything all day", "If someone doesn't come or make a phone call, and there are no chance of a conversation". In the interview after the start, there were most categories as "I came to think that vitality is important", "I became more motivated", and "I'm looking forward to talking".

Impression of conversation with robot: Verbatims were categorized as cute, funny, happy, humorous, exhilarating, exciting, pleasant, heartwarming, blissful, relaxing, relieved, and so on.

\section{DISCUSSION}

Brain training during the visits showed that there is a significant improvement in cognitive score, and that it is effective as a countermeasure for the elderly who cannot go out. Training performed at home one-on-one is more effective than group training. In one-on-one training, there is more flexibility to respond to various individual conditions and quickly react to a changing situation. Previous studies have also reported that individual training is more suited for individual abilities, and that responding to specific needs and preferences can meet the specific developmental needs of an individual [10-12].

In personal training sessions, the instructor can also focus $100 \%$ of their attention on the individual, enabling them to react in a safer and more appropriate manner. Elderly people who have difficulty going out are relatively frail, so this one-on-one training seems to be the best approach and their cognitive scores have improved significantly. If elderly people who have difficulties going out are left in their current situation, their mental and physical abilities will decrease.

Interviews carried out for this research also revealed a vicious cycle in which the less a person goes out, the more it becomes a bother to go out, and then even change clothes and adjust the appearance becomes a bother, and the person goes out even less. This situation exacerbates the decrease of abilities that are not being used. The interview also showed that there were days when a person would not talk 
with anyone all day, and the only opportunity for conversation was when someone came by or called them on the phone. Not talking to anyone all day is a big problem, and it has been confirmed that people who interact with others less than once per week have an about 1.4 times higher risk of getting dementia than people who interact with others every day [13].

People who have only limited social interactions are more likely to die early $[14,15]$, and have a low sense of purpose in life [16], high levels of anxiety [17], and a high risk of developing dementia [18]. Efforts to address this issue are lagging, and the only current solution is to rely on the help of volunteers.

In response to this problem, a robot can reduce labor shortage and greatly increase the amount of communication of elderly people. Encouraging conversation and interaction improves health [19-21], affects the length of the remaining life span [22], and prevents depression [23]. In addition, "pleasant emotions" such as cuteness and fun that the robot brings with it are associated with long life [24-26], influence the level of physical abilities [27], and prevent coronary artery disease [28,29]. However, elderly people who have difficulties going out have very few opportunities to experience pleasant emotions and it is difficult for them to change their mood.

Pets and such have become popular as a possible solution to improve a person's mood, but an apparent problem is that people cannot take care of pets anymore when they get older. Thus, if a robot could achieve the same ability to improve a person's mood and encourage feelings of attachment like a pet does, it can be expected to improve mood stability, reduce loneliness, reduce anxiety and stress [30], and improve cognitive function [31], along with other effects.

In conclusion, it was suggested that the interventions performed during a visit with the robot improved cognitive abilities as well as the mental health of elderly people with difficulties going out.

\section{CONCLUSION}

Brain training during the visits showed that there is a significant improvement in cognitive score, and that it is effective as a countermeasure for the elderly who cannot go out. In addition, the robot has a high affinity for all subjects, the effect of softening the tension associated with receiving the visit was confirmed.

Conversation with a robot that resolves the shortage of human resources is meaningful because in the situation where no one talks to anyone throughout the day the utilization of robot shows the improvement in energy and motivation due to increased conversation. By supplementing the interaction with regular supporters to this, it can be a more effective measure.

\section{ACKNOWLEDGMENT}

We would like to thank all the elderly people for participating in this project. In addition, we sincerely thank the members of support net for dementia prevention who cooperated. Furthermore, we want to thank everybody at Viston Corporation for their support in installing the brain training and rating scales into the robots.

\section{CONFLICT OF INTEREST}

All authors declare that they have no conflict of interest.

\section{FUNDING}

This research received assistance from the Japanese Ministry of Health, Labour and Welfare's dementia policy research fund.

\section{REFERENCES}

[1] National Institute of Population and Social Security Research. Status of connection and support between people. Survey on mutual support and life. 2013;17-25.

[2] Abe K. Dementia- Now, what you really want to know 101. Musashino University Press. 2017;1-232.

[3] Konno Y, Uesugi T. Examination of the Effects of Dohsa-Method Induced Positive Mind-Body Experience on Nostalgic Affects Using a EEG Pleasantness Scale and an Affective Imagery Scale. Human Sciences Research. 2003;25:63-72.

[4] Hayashi M, Saito H. How does nostalgia by music work on rest and calculations? Journal of Hokkaido University of Education. 2013;64:39-48.

[5] Al-Yahya E, Johansen-Berg $\mathrm{H}$, et al. Prefrontal cortex activation while walking under dual-task conditions in stroke: a multimodal imaging study. Neurorehabilitation and neural repair. 2016;30:591599.

[6] Ohsug H, Ohgi S, et al. Differences in dualtask performance and prefrontal cortex activation between younger and older adults. BMC neuroscience 2013;14:1-9.

[7] Anguera JA, Boccanfuso J, et al. Video game training enhances cognitive control in older adults. Nature. 2013;501: 97-101.

[8] Jaeggi SM, Studer-Luethi B, et al. The relationship between n-back performance and matrix reasoning implications for training and transfer. Intelligence. 2010;38: 625-635.

[9] McNab F, Varrone A, et al. Changes in cortical dopamine D1 receptor binding associated with cognitive training. Science. 2009;323: 800802 .

[10] Hirasawa K, Seki Y, et al. Effects of lifestyle improvement support centered on individual exercise programs. Shinshu Journal of Public Health. 2008;3:68-69.

[11] Jowett S, Kanakoglou K, et al. The application of the $3+1 \mathrm{Cs}$ relationship model in executive coaching. Consulting Psychology Journal: Practice and Research. 2012;64:183-197.

[12] Jackson, D. personal trainers can use self-efficacy theory to enhance exercise behavior in beginning exercisers. J Strength Cond Res. 2010;32:67-70.

[13] Saito M, Kondo K, et al. Different association between the loss of healthy life expectancy and social isolation by life satisfaction among older people : A four-year follow-up study of AGES project. Japanese Journal of Gerontology. 2013;35: 331-341.

[14] Aida J, Kondo K, et al. Assessing the association between all-cause mortality and multiple aspects of individual social capital among the older Japanese. BMC Public Health. 2011;25;1-16.

[15] Saito M, Kondo N, et al. Gender Differences on the Impacts of Social Exclusion on Mortality among Older Japanese : AGES Cohort Study. Social Science \& Medicine. 2012;75: 940-945.

[16] Cabinet Office. Survey on the willingness of the elderly to participate in the community. 2009 edition. Cabinet Office, Government of Japan. 2009:1-29.

[17] Kobayashi E, Fujiwara Y, et al. Social support availability and psychological well-being among the socially isolated elderly Differences by living arrangement and gender. Japanese Journal Of Public Health. 2011;58: 446-456.

[18] Saczynski J, Pfeifer L, et al. The effect of social engagement on incident dementia: the Honolulu-Asia Aging Study. American journal of epidemiology. 2006;163: 433-440.

[19] Martin L. Marital status, social capital, material conditions and selfrated health: A population-based study. Health Policy. 2009;93: 172179. 
[20] Gerry V, Isaac L, et al. Who you know, where you live : social capital, neighborhood and health. Social Science \& Medicine 2005;60:2799-2818

[21] Orma BE, Ruth W, et al. Individual-level analysis of social capital and health: A comparison of Arab and Jewish Israelis. Social Science \& Medicine 2008;66:900-910 .

[22] Anme T, Shinohara R, et al. Social interaction and mortality: A seven-year longitudinal study of elderly people. Japanese Journal Of Public Health. 2006;53: 681-687.

[23] Misawa J, Kondo K. Social factors relating to depression among older people in Japan: analysis of longitudinal panel data from the AGES project, Aging \& Mental Health. 2019;23: 1423-1432.

[24] Abel EL, Kruger ML. Smile intensity in photographs predicts longevity. Psychological Science, 2010;21: 542-544.

[25] Blazer DG, Hybels CF. What symptoms of depression predict mortality in community-dwelling

[26] elders? Journal of the American Geriatrics Society. 2004;52: 2052 2056.

[27] Moskowitz, JT, Epel ES, et al. Positive affect uniquely predicts lower risk of mortality in people with diabetes. Health Psychology. 2008;27: 573-582.

[28] Brummett $\mathrm{BH}$, Boyle $\mathrm{SH}$, et al. Positive affect is associated with cardiovascular reactivity, norepinephrine level, and morning rise in salivary cortisol. Psychophysiology. 2009;46: 862-869.

[29] Brummett $\mathrm{BH}$, Boyle $\mathrm{SH}$, et al. Ratings of positive and depressive emotion as predictors of mortality in coronary patients. International Journal of Cardiology. 2005;100: 213-216.

[30] Davidson KW, Mostofsky E, et al. Don't worry, be happy: positive affect and reduced 10-year incident coronary heart disease : The Canadian Nova Scotia Health Survey. European Heart Journal. 2010;31: 1065-1070.

[31] Ein N, Li L, et al. The effect of pet therapy on the physiological and subjective stress response: A meta-analysis. Stress Health. 2018;34: 477-489.

[32] Moretti F, Ronchi D, et al. Pet therapy in elderly patients with mental illness. The Official Journal of the Japanese Psychogeriatric Society. 2011;11: 125-129. 